\title{
Toward the Introduction of Google Assistant in Therapy for Children with Neurodevelopmental Disorders: An Exploratory Study
}

\author{
Fabio Catania* \\ fabio.catania@polimi.it \\ Politecnico di Milano \\ Milan, Italy
}

\author{
Micol Spitale* \\ micol.spitale@polimi.it \\ Politecnico di Milano \\ Milan, Italy
}

\author{
Franca Garzotto \\ Politecnico di Milano \\ Milan, Italy \\ franca.garzotto@polimi.it
}

\begin{abstract}
Despite the growing interest in Intelligent Personal Assistants in many domains, limited studies have explored this technology's usage among persons with neurodevelopmental disorders (NDD) and its potential for improving their cognitive skills. This paper presents an exploratory study that investigated the use of Google Home featured with Google Assistant in a therapeutic setting. We provided three therapists with two Google Home devices for 21 days, and they were welcome to use it as they liked at their ordinary oneto-one therapy sessions. During the study, we gathered different data: history logs from Google Assistant and semi-structured observations and comments by the specialists through questionnaires, forms, and a group interview. Our findings give a first glimpse of the usage patterns of Google Assistant within the therapy for children with NDD. Furthermore, our results point out the benefits and challenges for both therapists and children while interacting with conversational technology.
\end{abstract}

\section{CCS CONCEPTS}

- Human-centered computing $\rightarrow$ Empirical studies in HCI; • Social and professional topics $\rightarrow$ People with disabilities.

\section{KEYWORDS}

Intelligent Personal Assistants, Children, Neurodevelopmental Disorder, Disability

\section{ACM Reference Format:}

Fabio Catania, Micol Spitale, and Franca Garzotto. 2021. Toward the Introduction of Google Assistant in Therapy for Children with Neurodevelopmental Disorders: An Exploratory Study. In $\mathrm{CHI}$ Conference on $\mathrm{Hu}$ man Factors in Computing Systems Extended Abstracts (CHI '21 Extended Abstracts), May 8-13, 2021, Yokohama, Japan. ACM, New York, NY, USA, 7 pages. https://doi.org/10.1145/3411763.3451666

*Both authors contributed equally to this research.

Permission to make digital or hard copies of all or part of this work for personal or classroom use is granted without fee provided that copies are not made or distributed for profit or commercial advantage and that copies bear this notice and the full citation on the first page. Copyrights for components of this work owned by others than ACM must be honored. Abstracting with credit is permitted. To copy otherwise, or republish, to post on servers or to redistribute to lists, requires prior specific permission and/or a fee. Request permissions from permissions@acm.org.

CHI '21 Extended Abstracts, May 8-13, 2021, Yokohama, Japan

(c) 2021 Association for Computing Machinery.

ACM ISBN 978-1-4503-8095-9/21/05 . .\$15.00

https://doi.org/10.1145/3411763.3451666

\section{INTRODUCTION}

An Intelligent Personal Assistant (IPA) is a software agent that provides information by understanding human speech and responding via synthesized voice [54] (e.g., Siri, Alexa, Cortana, and Google Assistant [35]). IPAs are embedded into smartphones or dedicated home speakers and allow a voice-based hands-free interaction: users can use voice to ask them questions, control home automation devices, and manage other basic tasks such as email, to-do lists, and calendars [35]. In the last years, the Human-Computer Interaction community has been increasingly working on conversational technology $[10,23,31,41,45,51]$. Many studies have been carried out to understand how users adopt an IPA into their daily lives, and social interactions $[9,47,55]$ and to examine the perception of an IPA by users $[31,41,50]$. Also, much research has been done on IPAs to offer the potential to support the usage of groups with special needs, such as elderly [48, 60, 62], children [25], people with vision impairments [19], and military veterans with mild brain injury [57]. However, so far, there is still a limited understanding of how such conversational tools are used or could be used by people with neurodevelopmental disorders (NDD), especially by children. NDD, such as Autism Spectrum Disorder (ASD), is a group of conditions with onset in the developmental period. They typically produce low, medium or high severity levels of impairments in personal, social, academic, and occupational functioning [6]. Most forms of NDD are chronic, but early and focused interventions are thought to mitigate their effect $[20,32]$. From literature, we know that standard therapeutic interventions for children with NDD explore different approaches, such as play therapy [7], [34], cognitive behavior therapy [24, 36], and music therapy [58]. They aim to help children strengthen adaptive behaviors, develop stronger emotional and relational skills [7, 39], learn receptive and expressive language [27], and improve their self-care. In particular, the therapeutic tasks usually focus on developing: i) association skills; ii) emotional skills; iii) relational skills; iv) communicative and speech skills; v) abstract concepts (e.g., time and money). Recent research acknowledged interactive technology as a potentially useful tool to support standard interventions [4, 12, 37].

This work is an experimental study that explores the use of Google Assistant embedded into Google Home in a therapeutic setting with children with NDD. For 21 days, we provided three therapists with two Google Home devices, and they were welcome to use them as they wished at their regular one-to-one therapy sessions. We collected data from Google Assistant history logs and the specialists' observations through questionnaires, forms, and a semi-structured group interview. To the best of our knowledge, this 
paper describes the first exploratory study considering intelligent personal assistants for children with neurodevelopmental disorders in a therapeutic context. Please note that our emphasis is not on the therapy: this preliminary research is to be considered a premise for future investigations to understand the therapeutic value of conversational technologies for children with NDD. So far, we focused on the child-IPA and therapist-IPA interactions, and this paper is characterized by the following research goals:

RQ1. the identification of usage patterns with Google Assistant by therapists and children with NDD during therapy;

RQ2. the evaluation of their interaction benefits and challenges during interventions supported with Google Assistant.

\section{RELATED WORK}

The Human-Computer Interaction community has already addressed many studies involving conversational technologies and populations with special needs.

Some researchers investigated the design aspects of speech-based systems for elderly people [5, 48, 56, 60, 62]. For instance, Wulf et al. [60] focused on usability, user experience, and acceptance of speechbased interaction by the elderly and highlighted the potential of dialog systems for this target group. Other researchers focused on the IPAs for children [25, 44, 52]. Druga et al. [25] explored the use of different IPAs comparing Alexa, Google Assistant, Cozmo, and Julie Chatbot during child-agent interaction; they presented design considerations about voice and prosody, interactive engagement, and human-machine communication facilitation. Recently, few authors investigated the use of conversational agents for people with a mental, emotional, or physical disability, especially focusing on off-the-shelf commercial IPAs [3, 49]. Pradhan et al. [49] ran two studies in this domain: the first study analyzed 346 reviews of Amazon Echo, and a second study involved people with visual impairments. They concluded that despite some accessibility challenges, people with special needs can generally interact and use IPAs to complete specific tasks. Allen et al. [3] analyzed whether the Amazon Echo permitted the hands-free retrieval of visual supports for children with autism. Their findings showed that the autism target group generally struggled to use the Echo autonomously.

Also, the interest in supporting people with NDD through adhoc conversational agents is increasing in the HCI community $[14,16,17,61]$. However, to the best of our knowledge, so far, none explored the use of IPAs in an ecological environment to support therapy children with NDD. Our explorative study is the starting point to fill this gap.

\section{METHOD}

We conducted an exploratory study in a therapeutic center using two Google Home devices featured with Google Assistant. We asked therapists to use them during therapeutic sessions with children with NDD for three weeks. Therapists were free to use and let children use the assistant as they wished. As researchers, we never interacted with children but only with therapists before and after the study to respectively brief them and collect their feedback ${ }^{1}$.

\footnotetext{
${ }^{1}$ The authors certify that they have NO affiliations with or involvement in any organization or entity with any financial interest, or non-financial interest
}

\subsection{Participants}

The study involved 3 therapists and 9 children with NDD (see Table 1), including low, medium, and high severity from the therapeutic center SocioSfera. Participation was voluntary and restricted to users of the therapeutic centre. Children were among 4-17 y.o. and had been diagnosed with an ASD, or global developmental delay. C5, C7 had previously interacted with Google Assistant at home. The rest had no experience with any IPA. Children's personal data were processed in compliance with the provisions of current legislation on the protection of personal data. All parents signed an informed consent dealing with the experimental procedure acceptance and the use of data. The therapists (T1, T2, and $T 3)$ were recruited since they reported to be familiar with the technology in general, and they had already introduced technological devices (e.g., tablets) during therapy for children. However, none of them had any previous experience with any IPA yet. In this paper, we use the terms "participant/s" to include both therapists and children.

\subsection{Procedure}

The protocol of the study was approved by Politecnico di Milano ethics committee. The study lasted three weeks and took place in two rooms of the daycare center where children usually carry out their regular individual therapeutic session. Therapists placed the two IPAs on two tables (one for each therapy room), and they did not move the devices throughout the sessions. We configured Google Home with an ad-hoc-created Google account, enabled activity tracking, and also linked Google Assistant to a music service provider (i.e., Spotify). Before the study, we explained to the three therapists how to interact with Google Assistant by using the wake word "Ok Google" to trigger the system for any request, and the role of pause to mark the end of the speech. Also, we shared with the therapists the catalog of all features of Google Assistant as they are listed on the official website [1] (e.g., "Ok Google, play some music"). During sessions, therapists were completely free to use Google Assistant as they wished, and we did not provide them with any particular instruction or task to accomplish because we did not want to introduce any procedural bias.

\subsection{Data collection and analysis}

To gain multiple perspectives on how therapists and children with NDD used Google Assistant during their one-to-one therapeutic sessions, we engaged them in a complementary methods study involving the following:

- history logs. Google Assistant tracks the date, time, transcript, and response of all interactions by users;

- questionnaire. At the beginning of the study, we let therapists fill a short questionnaire to profile them. Questions aimed to discover the therapists' familiarity with commercial IPAs and their perception and expectations about the potential of IPAs for supporting children with NDD during therapy;

- forms. At the end of each therapeutic session, therapists filled a form regarding both the session in general and each of the specific tasks performed with Google Assistant. Open questions were about children's and therapists' usage of the agent, motivation for using the device within each activity, benefits/challenges/limitations concerning the conversational 
Table 1: Children involved in the exploratory study. The diagnosis nomenclature refers to DSM-V [6]

\begin{tabular}{cccccc}
\hline ID & Age & Gender & Verbal & Diagnosis & Severity Level \\
\hline C1 & 14 & $M$ & Yes & Autism & High \\
C2 & 5 & $M$ & $<10$ words & Autism & High \\
C3 & 9 & $M$ & No & Global Developmental delay & High \\
C4 & 5 & $M$ & Yes & Autism & Medium \\
C5 & 16 & $\mathrm{~F}$ & Yes & Autism & Low \\
C6 & 17 & M & Yes & Autism & Low \\
C7 & 16 & $\mathrm{~F}$ & Yes & Global Developmental delay & Low \\
C8 & 5 & $M$ & No & Autism & High \\
C9 & 4 & $M$ & $<10$ words & Autism & Medium \\
\hline
\end{tabular}

technology (including breakdowns), any recognized stereotype by children with NDD, automatic speech recognition experience, and suggestions for design improvement. Additionally, they scored children's initial engagement as they perceived it with a 1-10 likert scale;

- group interview [28]. At the end of the entire study, we interviewed the three therapists at a distance (via Google Meets). We opted for this data gathering method because the interaction between group specialists may give a more significant value and may arise different perspectives rather than just gathering their opinions individually [40]. We designed the interview to last one hour, and we used a semi-structured protocol. We asked therapists to tell us about the experience with the IPA during the study: the usage they made, the main activities they performed during the therapy, and the benefits and the challenges while interacting with the IPA. Therapists were also prompted to describe moments of joy and frustration with the conversational technology, moments in which they socially interacted with the device, and experiences of children with NDD interacting with it.

We opted not to directly interview children because questionbased approaches involve critical and self-critical skills [38] that are often lacking in these people [6]. Data analysis took inspiration from a previous study concerning Alexa and another group of people with special needs (i.e., people with visual impairments) [19]. Such as them, we analyzed separately quantitative data (i.e., history logs, part of the forms and the questionnaire) and qualitative data (i.e., the group interview, and part of the forms and the questionnaire) data, but we discussed them together.

We used a thematic coding approach [30] to analyze history logs. We identified the commands by participants linked by a common theme, and we indexed them into categories. Categories were not set a priori, but we obtained them with a bottom-up approach starting with simple themes and gradually impose meanings and connections in an inductive way. For example, the two utterances "Play today's top hits on Spotify" and "Play some reggae" could be included in the category "Play, or stop the music". Next, we ranked the categories based on the frequency of their requests.

About qualitative data, we grouped the responses and comments from the initial questionnaires, the forms, and the group interview depending on their topic $[13,21]$. Topics were set a priori in this case and were inspired by our research questions: (RQ1) usage patterns and (RQ2) participants' experience with IPA.

\section{FINDINGS}

Participants used Google Assistant during 17 therapy sessions in 21 days. All children had roughly the same number of sessions (1 or 2 each). From the history logs, questionnaires, forms and the group interview, it emerged how therapists and children with NDD interacted with the IPA in their regular therapy (RQ1), and which positive and negative experiences they had with the IPA (RQ2).

\subsection{Usage Patterns}

Therapists reported that children interacted alone with Google Assistant within 5 sessions, therapists alone for 7 sessions, and both children and therapists for 3 sessions. During only 2 sessions, children refused to interact with the assistant. Therapists told us that they integrated the IPA into their typical therapeutic activities. The activities varied in difficulty and type according to the subject. Generally, activities with Google Assistant were suggested by the therapist. Just once, $C 6$ spontaneously proposed to use the assistant to set the timer during a social game.History logs recorded in total 410 requests to the system. By applying the bottom-up thematic coding approach, we grouped them into 18 categories depending on the intention they convey (see Figure 1), named as usage pattern $[19,49]$. The most frequent requests were related to "Play animals' cry, or natural rumors" $(N=141)$, to "Play, or stop music" $(N=92)$, to set alarms or timers $(N=77)$, and to tune the volume $(N=28)$. Less frequently, participants used Google Assistant for asking the time $(N=12)$, playing built-in question-answer games $(N=11)$, asking for information $(N=10)$, and checking the weather $(N=7)$, small talk $(N=4)$, accessing the calendar $(N=3)$, getting news updates $(N=3)$, asking questions about interpersonal relationships (e.g., "How to greet a person when you meet her for the first time?") $(N=3)$ and about Google Assistant itself $(N=2$, i.e., "How are you?", "Where are you?"), translating sentences $(N=1)$, asking for the date $(N=1)$, cooking receipts $(N=1)$, and jokes $(N=1)$. In some cases, we could not identify the conversation intention (and consequently the category) from the commands' transcription $(N=11)$ due to the low accuracy of transcription or the lack of context information of the utterance (e.g., one transcription was "diagnose weights"). To examine if participants were equally interested in the 18 requests during therapy, we performed a one sample chi-square test. The frequency differences among categories were significant, $\chi^{2}(18)=1241.98, p<.001$. 


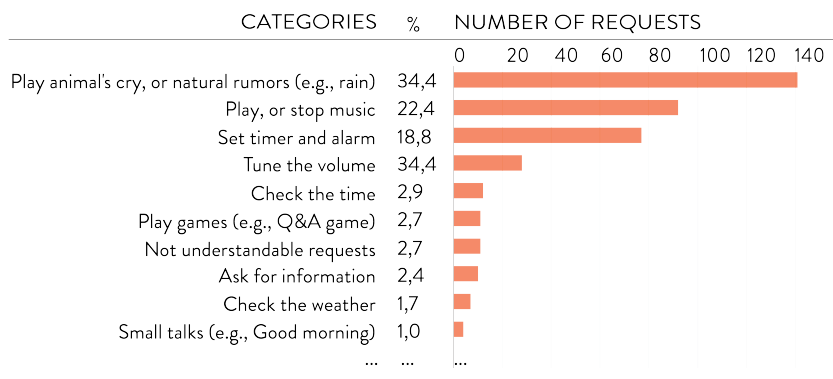

Figure 1: Usage pattern: the number of requests for each category (total number: $N=410$ )

\subsection{Children and therapists' experience with IPA}

From the thematic analysis of the forms, questionnaires, and the interview , three main topics emerged about the participants' experience with the IPA: children had some issues waking it up, and they encountered some barriers in interacting with it due to their cognitive, sensory, and language impairments. Nonetheless, therapists had an optimistic vision about introducing IPAs in therapy and they keep using Google Assistant even after the end of this study.

4.2.1 Waking up the IPA: children's challenges. To activate Google Assistant, users have to pronounce the wake word "Ok Google" and then add the request command (e.g., "Ok Google, set the timer for 10 minutes"). Therapists told us that this interaction was not trivial for children with NDD because they had difficulties in composing a sentence made by two parts of speech that are not semantically connected: the wake word and the request. For supporting them, therapists reported that they often mediated the children-assistant interaction: first, therapists triggered the IPA and then asked children to make the request. In addition, therapists reported that the full string (wake word and request) has been perceived as a slow interaction paradigm by children, because they often lost the attention and it was not easy for therapists to re-engage them to complete the request. To overcome the activation barriers and to meet children's particular needs, therapists proposed some interaction design solutions. First, instead of repeating the wake word at every conversational step, they would prefer to trigger the dialog system just once at the beginning of the interaction and let it active for a while. Then, they suggested using a more straightforward and direct wake word: they proposed "hey" instead of "Ok Google" because it contains less phonemes, so that it would be easier to pronounce without errors.In addition, learning "Ok Google" for starting a conversational interaction is not socially acceptable in any human-human interaction, while another wake work, such as "hey", can also be generalized in other social contexts. Third, therapists proposed hand-based trigger actions as valuable solutions, such clapping hands or pushing a button.

4.2.2 Children's language barriers, sensory fatigue, and adaptability. Therapists reported that the assistant misunderstood what participants asked in about 11 of the 17 sessions and sometimes it did not reply back at all. They attributed this dysfunctionality not only to technological limitations of the assistant but mostly to children's language impairments. The technological limitations of the automatic speech recognition system did not produce just negative results: some of the children enjoyed interacting with the IPA in autonomy despite not always being well understood. For example, $C 5$ tried to pronounce few words for 4 minutes in a row since she was motivated to improve her pronunciation. Therapists stated they wish to configure and customize already existing functionalities and contents offered by Google Assistant (e.g., its replies) to meet children's special needs. For example, both $C 2$ and $C 4$ did not recognize the sound of the timer because it was different from the one they were used to, and $T 1$ wished she could change it. Also, the repetition of the same standardized responses by the IPA caused stereotyped behaviors in some children. In fact, those with high severity often lost their attention and repeated over and over the last few words told by the assistant. Therapists highlighted the need of having a visual interface as a support to enable a more meaningful conversational experience for children with NDD. They suggested that the visual channel should be integrated with the speech to enable children to interact with different sensory modalities depending on their skills and attitudes. In fact, children with NDD often need to be stimulated from different sensory channels, otherwise they lack of sensory perception of the external world, loose their attention, and detach from the activity they are performing. Finally, therapists raised the need for children who had already interacted with Google Assistant at home to become accustomed to using it in the therapeutic setting as well. T1 explained that due their rigid mindset they associated the tool with a specific environment, such as home-context, and they were not able to extend it to other contexts.

4.2.3 Therapists' perception of the IPA and their vision. First, therapists reported that they easily learned how to communicate with Google Assistant since their very first interaction showing the intuitiveness of conversational technologies. They also found valuable the hands-free modality. It indeed enabled them to focus on the relationship with the child, rather than getting closer to the device (as happened when they used the tablet). Despite the usability barriers, therapists reported that the assistant supported children working on some relevant aspects for therapy (e.g., keeping the attention, turn-taking, respecting a communication protocol). Therapists wished they could create new activities for the therapy with the IPA, and personalize the already existing dialogues with Google Assistant adapting them to each single child.In this way, they could create a safer environment for children, let them work on their 
communication, social, and cognitive deficits, and have more control on the therapeutic tasks. Finally, they saw a great opportunity in recording therapeutic sessions and collecting information about children's behaviors to keep track of their progress. Regarding therapists perception of children initial engagement during the sessions, their general impression was positive, in fact they picked a score greater than 6 over 10 for 8 children over 9. In fact, they told that the majority of children were curious about the new device when they saw it in the room. Just two children were suspicious at the beginning and they did not want to interact with it. As soon as children got familiar with the assistant, most of them became even more interested than the first time. For the sake of completeness, therapists believed that children did not like Google Assistant itself, but they enjoyed the activities it enabled (e.g., playing with animals and listening to their cries). In addition, therapists also reported different interests towards the assistant between low and high severity children. High severity children did not ask for the Assistant unless the therapist proposed them an activity with it, while low severity children requested to interact with Google Assistant also when it wasn't prompted to do so.

\section{DISCUSSION}

Our findings highlight the role of IPAs integrated into therapeutic activities, and how IPAs can address the therapeutic needs. As chisquare test demonstrated, therapists decided to use some requests more frequently than others despite being furnished with a complete list of possible commands. We reported that the most frequent usage patterns involved playing some sounds as in previous studies with different populations [43, 47, 49, 56]. Indeed, during our 21-day study, therapists proposed activities (the animals' cries) to stimulate high severity children's association skills: children had to match visual stimuli (provided by means of conventional therapeutic materials such as images, cards, toys) with sound stimuli (generated by Google Assistant). In other activities, therapists used the IPA to play some music as a reward, reinforcement tool, relaxation [53], and to enhance listeners' social skills in turn-taking imitation, vocalization, and eye contact stimulation [26]. For the conceptualization and management of abstract concepts [15], our therapists introduced activities for tracking and managing time. This choice justifies the 77 requests about the time to Google Assistant (e.g., "Set the timer", "Check the time").

Although Google Assistant has somehow successfully supported the activities mentioned above, it failed with all those tasks to improve low severity children's communicative and socialization skills. To improve those abilities, therapists usually engage children in not just "short conversations" (based on simple, mutually independent request-response exchanges) but in "long conversations" (where the flow unfolds along mutually dependent conversation acts). Unlike previous studies on IPAs involving other populations where participants completed just "short conversations" with the IPA [43, 47, 49, 56], in our case, therapists attempted to trigger "long conversations" with Google Assistant [11]. They wanted to simulate typical dialogues that occur in everyday life during interpersonal relationships (e.g., "Small talks", "Asking questions about interpersonal relationships") to stimulate children with their social sphere. However, Google Assistant was not able to perform "long conversation" tasks as expected.

Also, the automatic speech recognition accuracy of Google Assistant resulted not high enough, especially when the speaker had some language impairments. The continuous misunderstanding could lead to frustration for the child, making the use of an IPA ineffective in therapeutic settings, as already reported in an analogous study on Amazon Echo for children with autism [3]. We agree with Beneteau et al. [8] that, if designers imbued voice assistants with the skills to identify that a communication partner has special needs, and therefore is likely to have imprecise speech and low discourse scaffolding skills, an intelligent personal assistant could provide more specific communication feedback to collaborate on the communication repair. Besides, since children often use repetition as their initial repair strategy in case of misunderstanding [46], it would be helpful for them and their therapist alike for assistants to provide a clear explanation on the reason why it is unable to answer properly, as already pinpointed in Druga et al. [25]. Indeed, if the issue was clear, therapists could help children employing different communication breakdown repair strategies depending on the context, such as defining terms [8]. We believe that integrating a visual communication channel could alleviate this issue as pinpointed by [59]. For example, Google Assistant could display the transcript of a user's speech so that people would know what the assistant understands. Besides, the visual channel could enhance the interaction, providing the users with visual feedback about the ongoing tasks (e.g., the running timer) to monitor their activities with the IPA. Therapists also wish to see integrated in the next generation IPAs are the straightforward customization of tasks and dialogues and the automated analysis of interaction data analysis to monitor children's progress. Also, to make Google Assistant more adoptable during therapy, the IPA should be more accessible and autonomously usable by people with NDD. Interaction designers might consider using different wake words or even a new wake action. Alternatives could be of a different nature (vocal, tactile, visual, event-based, motion-based). A previous study [18] sheds light on the importance of physicality for children's interaction even speaking about conversational interfaces, and suggests the usage of a physical button to wake up and put to sleep the conversational agent. Catania et al. [18] proposed to use identical actions both to wake up and to put to sleep the IPA, and provide a theoretical argument based on the theory of partner-perceived communication $[22,42]$, which states that the predictability and repetitiveness of the sequences make it possible to better give meaning to them even for those children with complex communication needs.

\subsection{Limitations}

This paper's contribution is limited due to a highly exploratory approach focusing on the therapist-IPA and child-IPA interactions and with little emphasis on the possible support that Google Assistant may have as part of a therapeutic regime. The study took place in a limited period (21 days) and, consequently, only preliminary insights emerged and not a complete overview of the valuable features for the adoption of Google Assistant in a therapeutic context. For instance, Garg and Sengupta [29] found out that neuro-typical 
children interacting with IPAs reduced their communication breakdowns substantially over the long-term. We are aware that with specially-abled children handling communication breakdowns might be a more significant challenge, but still, some of them could learn the conversational interaction paradigm with time. Also, another limitation of the study is that the sample we involved ( 3 therapists and nine children) was small, potentially impacting the generalization of the findings for the whole population. As a means of defense, it should be noticed that our study is in line with previous ones on people with special needs both in terms of duration (e.g., [19]) and sample size (e.g., [2, 33]). Finally, therapists reported that the interaction with the IPA would be smoother for children with NDD by using visual support. We are aware that Google Assistant enables it via smartphones, tablets, and Google Nest. However, we did not opt for those solutions because of affordability reasons.

\section{CONCLUSION}

This paper described an empirical 21-day study investigating how 3 therapists and 9 children with NDD interacted with the IPA Google Assistant during their regular individual therapy sessions. Findings showed that Google Assistant has the potential for being used as a stimulus for oral communication and socialization in many activities with children from low to high severity. However, children's cognitive, sensory, and language impairments make it difficult for them to autonomously use Google Assistant. They cannot be easily understood by the automatic speech recognition system, and they cannot quickly adapt to the schematic communicative protocol involving the use of the wake word. We conclude that Google Assistant could be considered a tool for therapists, who may decided to invite children to use it.

Our preliminary results provide interaction designers and conversational technology developers with new insights about Google Assistant (and IPAs in general) interacting with children with special needs. Furthermore, our research findings might pave the ground towards new forms of therapeutic interventions for children with NDD that exploit conversational agents within existing practices. Such therapies will be the subject of our follow-up study.

\section{REFERENCES}

[1] [n.d.]. Google Assistant. https://assistant.google.com/explore?hl=en_us

[2] Mohammad Rafayet Ali, Zahra Razavi, Abdullah Al Mamun, Raina Langevin, Reza Rawassizadeh, Lenhart Schubert, and M Ehsan Hoque. 2018. A Virtual Conversational Agent for Teens with Autism: Experimental Results and Design Lessons. arXiv preprint arXiv:1811.03046 (2018).

[3] Anna A Allen, Howard C Shane, and Ralf W Schlosser. 2018. The Echo ${ }^{\mathrm{TM}}$ as a speaker-independent speech recognition device to support children with autism: an exploratory study. Advances in Neurodevelopmental Disorders 2, 1 (2018), 69-74.

[4] Gordon Alley-Young. 2016. Technology tools for students with autism: innovations that enhance independence and learning. Canadian fournal of Communication 41, 3 (2016)

[5] Leonardo Angelini, Maurizio Caon, Stefano Carrino, Luc Bergeron, Nathalie Nyffeler, Mélanie Jean-Mairet, and Elena Mugellini. 2013. Designing a desirable smart bracelet for older adults. In Proceedings of the 2013 ACM conference on Pervasive and ubiquitous computing adjunct publication. 425-434.

[6] American Psychiatric Association et al. 2013. Diagnostic and statistical manual of mental disorders (DSM-5®). American Psychiatric Pub.

[7] Randall L Astramovich, Catherine Lyons, and Nancy J Hamilton. 2015. Play therapy for children with intellectual disabilities. Fournal of Child and Adolescent Counseling 1, 1 (2015), 27-36.

[8] Erin Beneteau, Olivia K Richards, Mingrui Zhang, Julie A Kientz, Jason Yip, and Alexis Hiniker. 2019. Communication Breakdowns Between Families and Alexa. In Proceedings of the 2019 CHI Conference on Human Factors in Computing
Systems (Glasgow, Scotland Uk) (CHI '19, Paper 243). Association for Computing Machinery, New York, NY, USA, 1-13.

[9] Frank Bentley, Chris Luvogt, Max Silverman, Rushani Wirasinghe, Brooke White, and Danielle Lottridge. 2018. Understanding the Long-Term Use of Smart Speaker Assistants. Proc. ACM Interact. Mob. Wearable Ubiquitous Technol. 2, 3, Article 91 (Sept. 2018), 24 pages. https://doi.org/10.1145/3264901

[10] Apoorva Bhalla. 2018. An Exploratory Study Understanding the Appropriated Use of Voice-Based Search and Assistants. In Proceedings of the 9th Indian Conference on Human Computer Interaction (Bangalore, India) (IndiaHCI'18). Association for Computing Machinery, New York, NY, USA, 90-94. https://doi.org/10.1145/ 3297121.3297136

[11] Timothy Bickmore and Justine Cassell. 2005. Social dialongue with embodied conversational agents. In Advances in natural multimodal dialogue systems. Springer, 23-54.

[12] Emily C Bouck, Melissa Savage, Nancy K Meyer, Teresa Taber-Doughty, and Megan Hunley. 2014. High-tech or low-tech? Comparing self-monitoring systems to increase task independence for students with autism. Focus on Autism and Other Developmental Disabilities 29, 3 (2014), 156-167.

[13] Virginia Braun and Victoria Clarke. 2006. Using thematic analysis in psychology. Qualitative research in psychology 3, 2 (2006), 77-101.

[14] Stéphanie Buisine and Jean-Claude Martin. 2005. Children's and adults' multimodal interaction with 2D conversational agents. In CHI'05 Extended Abstracts on Human Factors in Computing Systems. 1240-1243.

[15] Martin Casassus, Ellen Poliakoff, Emma Gowen, Daniel Poole, and Luke Anthony Jones. 2019. Time perception and autistic spectrum condition: A systematic review. Autism Research 12, 10 (2019), 1440-1462.

[16] Justine Cassell. 2001. Embodied conversational agents: representation and intelligence in user interfaces. AI magazine 22, 4 (2001), 67-67.

[17] Fabio Catania, Nicola Di Nardo, Franca Garzotto, and Daniele Occhiuto. 2019. Emoty: an emotionally sensitive conversational agent for people with neurodevelopmental disorders. In Proceedings of the 52nd Hawaii International Conference on System Sciences.

[18] Fabio Catania, Micol Spitale, Giulia Cosentino, and Franca Garzotto. 2020. What is the Best Action for Children to "Wake Up" and "Put to Sleep" a Conversational Agent? A Multi-Criteria Decision Analysis Approach. In Proceedings of the 2nd Conference on Conversational User Interfaces (Bilbao, Spain) (CUI '20). Association for Computing Machinery, New York, NY, USA, Article 4, 10 pages. https: //doi.org/10.1145/3405755.3406129

[19] Dasom Choi, Daehyun Kwak, Minji Cho, and Sangsu Lee. 2020. " Nobody Speaks that Fast!" An Empirical Study of Speech Rate in Conversational Agents for People with Vision Impairments. In Proceedings of the 2020 CHI Conference on Human Factors in Computing Systems. 1-13.

[20] G. Cioni, E. Inguaggiato, and G. Sgandurra. 2016. Early intervention in neurodevelopmental disorders: underlying neural mechanisms. Developmental Medicine \& Child Neurology 58 (2016).

[21] Harris Ed Cooper, Paul M Camic, Debra L Long, AT Panter, David Ed Rindskopf, and Kenneth J Sher. 2012. APA handbook of research methods in psychology, Vol 2: Research designs: Quantitative, qualitative, neuropsychological, and biological. (2012).

[22] M. A. Costantino. 2011. Costruire libri e storie con la CAA: gli IN-Books per l'intervento precoce e linclusione. Erickson.

[23] Benjamin R. Cowan, Nadia Pantidi, David Coyle, Kellie Morrissey, Peter Clarke, Sara Al-Shehri, David Earley, and Natasha Bandeira. 2017. "What Can i Help You with?": Infrequent Users' Experiences of Intelligent Personal Assistants. In Proceedings of the 19th International Conference on Human-Computer Interaction with Mobile Devices and Services (Vienna, Austria) (MobileHCI '17). Association for Computing Machinery, New York, NY, USA, Article 43, 12 pages. https: //doi.org/10.1145/3098279.3098539

[24] John T Danial and Jeffrey J Wood. 2013. Cognitive behavioral therapy for children with autism: review and considerations for future research. Fournal of Developmental \& Behavioral Pediatrics 34, 9 (2013), 702-715.

[25] Stefania Druga, Randi Williams, Cynthia Breazeal, and Mitchel Resnick. 2017. "Hey Google is It OK If I Eat You?": Initial Explorations in Child-Agent Interaction. In Proceedings of the 2017 Conference on Interaction Design and Children (Stanford, California, USA) (IDC '17). Association for Computing Machinery, New York, NY, USA, 595-600. https://doi.org/10.1145/3078072.3084330

[26] Barbara Duffy and Ray Fuller. 2000. Role of music therapy in social skills development in children with moderate intellectual disability. Fournal of Applied Research in Intellectual Disabilities 13, 2 (2000), 77-89.

[27] Michelle Flippin, Stephanie Reszka, and Linda R Watson. 2010. Effectiveness of the Picture Exchange Communication System (PECS) on communication and speech for children with autism spectrum disorders: A meta-analysis. American Journal of Speech-Language Pathology (2010).

[28] James H Frey and Andrea Fontana. 1991. The group interview in social research. The Social Science fournal 28, 2 (1991), 175-187.

[29] Radhika Garg and Subhasree Sengupta. 2020. He Is Just Like Me: A Study of the Long-Term Use of Smart Speakers by Parents and Children. Proc. ACM Interact. Mob. Wearable Ubiquitous Technol. 4, 1 (March 2020), 1-24. 
[30] G R Gibbs. 2007. Thematic coding and categorizing. (2007)

[31] Leilani H. Gilpin, Danielle M. Olson, and Tarfah Alrashed. 2018. Perception of Speaker Personality Traits Using Speech Signals. In Extended Abstracts of the 2018 CHI Conference on Human Factors in Computing Systems (Montreal OC, Canada) (CHI EA '18). Association for Computing Machinery, New York, NY, USA, 1-6. https://doi.org/10.1145/3170427.3188557

[32] Michael J Guralnick. 2011. Why early intervention works: A systems perspective Infants and young children 24, 1 (2011), 6.

[33] S Grace Tinnunnem Haokip, Griva Shah, and Uttama Lahiri. 2017. Psychophysiological implications of computer based social and non-social interactive tasks for children with autism. In 2017 8th International Conference on Computing, Communication and Networking Technologies (ICCCNT). IEEE, 1-7.

[34] Heidi Hillman. 2018. Child-centered play therapy as an intervention for children with autism: A literature review. International Journal of Play Therapy 27, 4 (2018), 198.

[35] Matthew B Hoy. 2018. Alexa, Siri, Cortana, and more: an introduction to voice assistants. Medical reference services quarterly 37, 1 (2018), 81-88.

[36] Anastasia Hronis, Lynette Roberts, and Ian I Kneebone. 2017. A review of cognitive impairments in children with intellectual disabilities: Implications for cognitive behaviour therapy. British fournal of Clinical Psychology 56, 2 (2017), 189-207.

[37] Muhammad Shoaib Jaliaawala and Rizwan Ahmed Khan. 2019. Can autism be catered with artificial intelligence-assisted intervention technology? A comprehensive survey. Artificial Intelligence Review 53 (2019), 1039-1069.

[38] Patrick W Jordan, Bruce Thomas, Ian Lyall McClelland, and Bernard Weerdmeester. 1996. Usability evaluation in industry. CRC Press.

[39] Jinah Kim, Tony Wigram, and Christian Gold. 2009. Emotional, motivational and interpersonal responsiveness of children with autism in improvisational music therapy. Autism 13, 4 (2009), 389-409.

[40] Richard A Krueger. 2014. Focus groups: A practical guide for applied research. Sage publications

[41] Sunok Lee, Sungbae Kim, and Sangsu Lee. 2019. "What Does Your Agent Look like?": A Drawing Study to Understand Users' Perceived Persona of Conversational Agent. In Extended Abstracts of the 2019 CHI Conference on Human Factors in Computing Systems (Glasgow, Scotland Uk) (CHI EA '19). Association for Computing Machinery, New York, NY, USA, 1-6. https://doi.org/10.1145/3290607.3312796

[42] Janice Light and Kathryn Drager. 2007. AAC technologies for young children with complex communication needs: State of the science and future research directions. Augmentative and alternative communication 23, 3 (2007), 204-216.

[43] Irene Lopatovska and Harriet Williams. 2018. Personification of the Amazon Alexa: BFF or a mindless companion. In Proceedings of the 2018 Conference on Human Information Interaction \& Retrieval. 265-268.

[44] Silvia B Lovato, Anne Marie Piper, and Ellen A Wartella. 2019. Hey Google, Do Unicorns Exist? Conversational Agents as a Path to Answers to Children's Questions. In Proceedings of the 18th ACM International Conference on Interaction Design and Children. 301-313.

[45] Ewa Luger and Abigail Sellen. 2016. "Like Having a Really Bad PA": The Gulf between User Expectation and Experience of Conversational Agents. In Proceedings of the 2016 CHI Conference on Human Factors in Computing Systems (San Jose, California, USA) (CHI '16). Association for Computing Machinery, New York, NY, USA, 5286-5297. https://doi.org/10.1145/2858036.2858288

[46] Tova Most. 2002. The Use of Repair Strategies by Children With and Without Hearing Impairment. Lang. Speech Hear. Serv. Sch. 33, 2 (April 2002), 112-123.

[47] Martin Porcheron, Joel E. Fischer, Stuart Reeves, and Sarah Sharples. 2018. Voice Interfaces in Everyday Life. In Proceedings of the 2018 CHI Conference on Human Factors in Computing Systems (Montreal QC, Canada) (CHI '18). Association for Computing Machinery, New York, NY, USA, 1-12. https://doi.org/10.1145/ 3173574.3174214

[48] François Portet, Michel Vacher, Caroline Golanski, Camille Roux, and Brigitte Meillon. 2013. Design and evaluation of a smart home voice interface for the elderly: acceptability and objection aspects. Personal and Ubiquitous Computing 17, 1 (2013), 127-144.

[49] Alisha Pradhan, Kanika Mehta, and Leah Findlater. 2018. " Accessibility Came by Accident" Use of Voice-Controlled Intelligent Personal Assistants by People with Disabilities. In Proceedings of the 2018 CHI Conference on Human Factors in Computing Systems. 1-13.

[50] Amanda Purington, Jessie G. Taft, Shruti Sannon, Natalya N. Bazarova, and Samuel Hardman Taylor. 2017. "Alexa is My New BFF": Social Roles, User Satisfaction, and Personification of the Amazon Echo. In Proceedings of the 2017 CHI Conference Extended Abstracts on Human Factors in Computing Systems (Denver, Colorado, USA) (CHI EA '17). Association for Computing Machinery, New York, NY, USA, 2853-2859. https://doi.org/10.1145/3027063.3053246

[51] Aung Pyae and Tapani N. Joelsson. 2018. Investigating the Usability and User Experiences of Voice User Interface: A Case of Google Home Smart Speaker. In Proceedings of the 20th International Conference on Human-Computer Interaction with Mobile Devices and Services Adjunct (Barcelona, Spain) (MobileHCI '18). Association for Computing Machinery, New York, NY, USA, 127-131. https: //doi.org/10.1145/3236112.3236130

[52] Marie Radford, Diana Floegel, Sarah Barriage, and Daniel Houli. 2019. "Alexa, where do babies come from?" Investigating children's practices with intelligent personal assistants. User interfaces (2019).

[53] Alaine E Reschke-Hernández. 2011. History of music therapy treatment interventions for children with autism. Fournal of Music Therapy 48, 2 (2011), 169-207.

[54] Joao Santos, Joel JPC Rodrigues, João Casal, Kashif Saleem, and Victor Denisov. 2016. Intelligent personal assistants based on internet of things approaches. IEEE Systems fournal 12, 2 (2016), 1793-1802.

[55] Alex Sciuto, Arnita Saini, Jodi Forlizzi, and Jason I. Hong. 2018. "Hey Alexa, What's Up?": A Mixed-Methods Studies of In-Home Conversational Agent Usage. In Proceedings of the 2018 Designing Interactive Systems Conference (Hong Kong, China) (DIS '18). Association for Computing Machinery, New York, NY, USA, 857-868. https://doi.org/10.1145/3196709.3196772

[56] Milka Trajkova and Aqueasha Martin-Hammond. 2020. " Alexa is a Toy": Exploring Older Adults' Reasons for Using, Limiting, and Abandoning Echo. In Proceedings of the 2020 CHI Conference on Human Factors in Computing Systems. $1-13$.

[57] T Wallace and J Morris. 2018. Identifying barriers to usability: smart speaker testing by military veterans with mild brain injury and PTSD. In Cambridge Workshop on Universal Access and Assistive Technology. Springer, 113-122.

[58] Auriel Warwick and Juliette Alvin. 1991. Music therapy for the autistic child. Oxford University Press.

[59] Yunhan Wu, Daniel Rough, Anna Bleakley, Justin Edwards, Orla Cooney, Philip R Doyle, Leigh Clark, and Benjamin R Cowan. 2020. See What I'm Saying? Comparing Intelligent Personal Assistant Use for Native and Non-Native Language Speakers. In 22nd International Conference on Human-Computer Interaction with Mobile Devices and Services. 1-9.

[60] Linda Wulf, Markus Garschall, Julia Himmelsbach, and Manfred Tscheligi. 2014. Hands Free - Care Free: Elderly People Taking Advantage of Speech-Only Interaction. In Proceedings of the 8th Nordic Conference on Human-Computer Interaction: Fun, Fast, Foundational (Helsinki, Finland) (NordiCHI '14). Association for Computing Machinery, New York, NY, USA, 203-206. https://doi.org/10.1145/2639189. 2639251

[61] Ying Xu and Mark Warschauer. 2019. Young children's reading and learning with conversational agents. In Extended Abstracts of the 2019 CHI Conference on Human Factors in Computing Systems. 1-8.

[62] Randall Ziman and Greg Walsh. 2018. Factors Affecting Seniors' Perceptions of Voice-Enabled User Interfaces. In Extended Abstracts of the 2018 CHI Conference on Human Factors in Computing Systems (Montreal QC, Canada) (CHI EA '18). Association for Computing Machinery, New York, NY, USA, 1-6. https://doi. org $/ 10.1145 / 3170427.3188575$ 